\title{
Time-varying Resilient Virtual Network Mapping for Multi-location Cloud Data Centers
}

\author{
Minh Bui ${ }^{1}$, Ting Wang ${ }^{1}$, Brigitte Jaumard ${ }^{1}$, Deep Medhi ${ }^{2}$, Chris Develder $^{3}$ \\ ${ }^{1}$ CSE, Concordia University, Montreal (Qc) H3G $1 M 8$ Canada \\ ${ }^{2}$ CSEE, University of Missouri, Kansas City, MO, USA \\ ${ }^{3}$ INTEC - IBCN, Ghent University - iMinds, Ghent, Belgium
}

\begin{abstract}
Optical networks constitute a fundamental building block that has enabled the success of cloud computing. Virtualization, a cornerstone of cloud computing, today is applied in the networking field: physical network infrastructure is logically partitioned into separate virtual networks, thus providing isolation between distinct virtual network operators (VNOs). Hence, the problem of virtual network mapping has arisen: how to decide which physical resources to allocate for a particular virtual network? In a cloud context, not just network connectivity is required, but also data center (DC) resources located at multiple locations, for computation and/or storage. Given the underlying anycast routing principle, the network operator has some freedom to which specific DC to allocate these resources.

In this paper, we solve a resilient virtual network mapping problem that optimally decides on the mapping of both network and multi-location data center resources resiliently using anycast routing, considering time-varying traffic conditions. In terms of resilience, we consider the so-called VNO-resilience scheme, where resilience is provided in the virtual network layer. To minimize physical resource capacity requirements, we allow reuse of both network and DC resources. The failures we protect against include both network and DC resource failures: we hence allocate backup DC resources, and also account for synchronization between primary and backup DC.

As optimization criteria, we not only consider resource usage minimization, but also aim to limit virtual network reconfigurations from one time period to the next. We propose a scalable column generation approach to solve the dynamic resilient virtual network mapping problem, and demonstrate it in a case study on a nationwide US backbone network.
\end{abstract}

Keywords: Network Virtualization, End-to-End Resilience, Cloud Computing, Anycast Resilience.

\section{Introduction}

The survivability of optical networks, to support cloud services (distributed over multiple locations), is a critical concern. While shared protection schemes allow significant bandwidth saving, additional saving can be achieved through reconfiguration whenever the traffic is highly time-varying. We consider a timeslotted approach, where the traffic requests change from one time period to the next, and investigate the usefulness of reconfiguring the traffic routes when a new time slot starts. Such reconfiguration may involve changing working and/or backup paths for (some of) the traffic flows. Since changing the working path of ongoing traffic might be too disruptive (or unacceptable for some time-critical, high QoS services), we investigate also the potential benefit (in terms of overal reduced link bandwidth occupancy) of only modifying the backup paths. We aim at quantifying the maximal bandwidth savings with a minimal number of path routing changes for traffic that continues from one period to the next. We solve a resilient virtual network mapping problem to optimally decide on the mapping of both network and multi-location data center resources resiliently using anycast routing, under time-varying traffic conditions.

This topic has been investigated in the past, but not thoroughly. For instance, He and Poo [1] propose a sub-reconfiguration technique in order to rearrange the paths for WDM (Wavelength Division Multiplexing) networks, using pre-computed alternate backup paths. They report a $10 \%$ bandwidth saving with simulation experiments using OPNET. Other studies look at differentiated protection schemes, e.g., [2] or [3], with either pre-emption or multiple protection paths, but without backup reconfiguration.

In the context of optical grids and cloud computing, the design of resilient networks has been studied within the anycast framework, see, e.g., [4,5]. However, we are not aware of any work on time-varying anycast traffic exploiting protection rerouting or reconfiguration.

The paper is organized as follows. In Section 2, we discuss the resilience issues in cloud computing 
where the physical infrastructure is usually shared by multiple virtual network operators (VNOs) and we explain the issues raised by time-varying traffic for minimizing the backup bandwidth requirements. In Section 3, we propose a new model for investigating various scenarios of primary or backup disruption in order to minimize the bandwidth requirements in the context of dynamic traffic. Results are reported in Section 5. Conclusions are drawn in the final Section 6.

\section{Problem Statement}

\subsection{Virtualization and Resilience in Cloud Computing}

The recent evolution towards grid and cloud computing illustrates the crucial role played by (optical) networks in supporting today's applications [6]. A core concept in cloud computing is that of virtualization: an extra layer of abstraction is provided, such that the same physical infrastructure can be simultaneously used by distinct entities, each running their own applications in a virtually isolated environment. This allows more efficient use of the physical infrastructure, as well as fexible extension of capacity by adding more virtual machines (and distributing them among multiple physical machines). The same idea of virtualization is also applied in the networking domain [7]: physical infrastructure (i.e., fibers and optical cross-connects, OXCs, ROADMs) can be shared by multiple virtual network operators (VNOs), who only see their own resources in a virtual topology, and have full control over it. Combining both network and server virtualization in the optical cloud calls for joint optimized provisioning mechanisms allocating both network and IT resources [8].

In this paper, we consider the physical network and data center resources to be owned and operated by physical infrastructure providers (PIPs; note that the PIP for data center resources may be a different entity than the PIP for the optical network). The cloud services' requests are offered by a virtual network operator (VNO), which runs its VNet on top of the PIP resources. The problem we address is how to determine a resilient VNet topology that minimizes the bandwidth resources that are requested by the VNO to the PIP, assuming time-varying traffic. We assume a VNO-resilience scheme, i.e., rerouting in the virtual network under the VNO control (see below, Section 2.2, or, e.g., [5]).

Cloud services' requests are characterized by their origin $s$ (i.e., the location of customer of the VNO), and need to be served at a data center $d$ (where server capacity should be allocated) and requires network connectivity between the $(s, d)$ pair. Assuming anycast, $d$ can be chosen out of a set of given locations (i.e., where the VNO can rely on a PIP's infrastructure). We design the VNet such that requests can survive single failures, which can each affect either the physical network or data center infrastructure.

\subsection{VNO-resilience}

As illustrated in Fig. 1, the VNO-resilience model provides 1:1 protection routing in the VNet for network failures, where the working and protection paths of a service have to be physically link/node disjoint: the working path $\left(p^{\mathrm{w}}\right)$ routes the services towards the primary DC, the protection path $\left(p^{\mathrm{B}}\right)$ towards the backup DC, while $p^{\mathrm{W}}$ and $p^{\mathrm{B}}$ are disjoint in their physical layer mapping. In addition, a synchronization path $\left(p^{\mathrm{S}}\right)$ is established in order to handle migration and failure routing requirements when a DC failure occurs: services then need to be rerouted from the primary $d_{1}$ to backup $d_{2}$. Thus, the resulting VNet for the request from source $v_{s}$ comprises three virtual paths (comprising 5 virtual links in total, in this example), mapped to resp. the physical $p^{\mathrm{W}}, p^{\mathrm{B}}$ and $p^{\mathrm{S}}$ paths. Note that both $p^{\mathrm{W}}$ and $p^{\mathrm{B}}$ need to carry the overall traffic (but $p^{\mathrm{B}}$ only when $p^{\mathrm{W}}$ or $d_{1}$ are affected by a failure), but $p^{\mathrm{S}}$ possibly only a fraction thereof, only to keep the state at the backup location $d_{2}$ synchronized with that of $d_{1}$ (or vice versa) to allow smooth migration upon $d_{1}$ failure (or recovery).

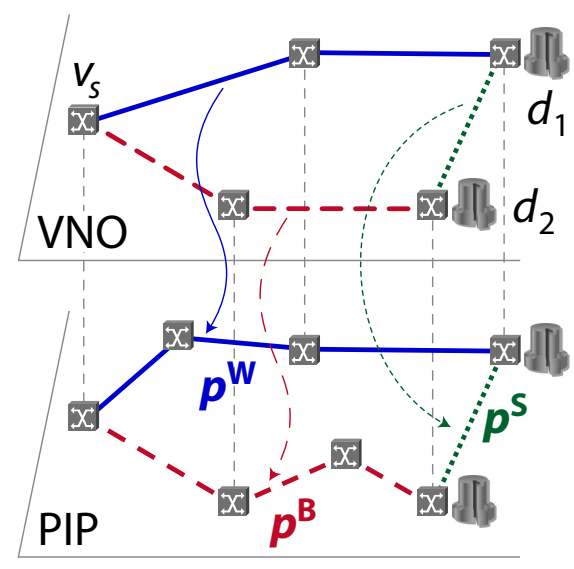

Fig. 1: The VNO-resilience scheme. 
Further, we assume that there is an automatic switch-back to the original network path or DC once a fault is repaired, and therefore we allow reusing the same network/DC capacity to protect against other failures: backup capacity is shared. Under the assumptions that (A1) the backup DC has a different location than the primary $\mathrm{DC},(\mathrm{A} 2) p^{\mathrm{W}}$ and $p^{\mathrm{B}}$ are link disjoint and, (A3) $p^{\mathrm{W}}$ and $p^{\mathrm{S}}$ are link disjoint, protection is guaranteed against any single link failure and any single DC failure. We now qualitatively discuss the various failure cases we protect against:

(i) Failure of link $\ell \in p^{\mathrm{w}}$ : the request is rerouted to the backup data center $d_{1}$, using the backup path $p^{\mathrm{B}}$ (which is link disjoint from $p^{\mathrm{W}}$, thus $\ell \notin p^{\mathrm{B}}$ ). If $\ell \in p^{\mathrm{S}} \cap p^{\mathrm{W}}$, then as long as the failure is not restored, the primary data center $d_{1}$ cannot be kept in sync with the now operational $d_{2}$. Thus, right after the repair of $\ell$, the primary $d_{1}$ is in stale state, and hence switching back to $d_{1}$ either suffers from this stale state or needs to wait some extra time to handle the requests again. The remedy is of course to enforce $p^{\mathrm{W}} \cap p^{\mathrm{S}}=\emptyset$. (Yet, note that the same issue of a non-synchronized primary $d_{1}$ clearly also occurs after the repair of $d_{1}$ that failed itself.)

(ii) Failure of link $\ell \in p^{\mathrm{S}} \backslash p^{\mathrm{w}}$ : there is no immediate issue. Yet, if shortly after $\ell$ 's repair, working path $p^{\mathrm{W}}$ fails, the switchover to the backup $d_{2}$ (via path $p^{\mathrm{B}}$ ) suffers from stale state since the failing $p^{\mathrm{S}}$ interrupted the synchronization between primary and backup DCs. This can only be remedied by providing a second synchronization path $p^{\text {sS }}$ that is link disjoint with $p^{\mathrm{S}}$.

(iii) Failure of link $\ell \in p^{\mathrm{B}}$ : again no immediate problem arises (since this means that $p^{\mathrm{W}}$ is operational, given $p^{\mathrm{W}} \cap p^{\mathrm{B}}=\emptyset$ ). However, if $\ell \in p^{\mathrm{S}} \cap p^{\mathrm{B}}$ and shortly after $\ell^{\prime}$ 's repair the primary path $p^{\mathrm{W}}$ (or $d_{1}$ ) fails - meaning that now B is followed towards $d_{2}$ - the secondary data center $d_{2}$ might not be fully sync'ed yet. Clearly, this can be remedied by choosing $p^{\mathrm{B}} \cap p^{\mathrm{S}}=\emptyset$. Yet, the issue is similar to the one of case (ii), which obviously remains, even if we take $p^{\mathrm{S}} \cap p^{\mathrm{B}}=\emptyset$.

(iv) Failure of primary DC $d_{1}$ : requests are rerouted to backup $d_{2}$ via the $p^{\mathrm{B}}$ path. Clearly, the failing $d_{1}$ cannot be kept in sync with the now operational backup $d_{2}$. Thus, we might need to wait some time after $d_{1}$ 's repair to switch back requests via $p^{\mathrm{W}}$. Any failure that would occur shortly after $d_{1}$ 's repair and which would prevent services to remain being served at $d_{2}$ clearly could imply service degradation because of the unsynchronized $d_{1}$ : (a) failure of $p^{\mathrm{S}}$, (b) failure of $p^{\mathrm{B}}$, or (c) failure of $d_{2}$. However, protection against such a failure event requires extra DC resources or extra paths.

\subsection{Time-varying Traffic}

The motivation of this study is to investigate whether it is worth reconfiguring the primary and the backup paths in order to save bandwidth when the communication traffic pattern changes. Note that this change is not necessarily limited to a scaling of the volume, but also its geographical pattern: when considering large backbone networks (as the ones that we are designing VNets over), they might comprise different time zones where activities are shifted in time, and hence the resulting volume of cloud requests fluctuates differently.

As changing the VNet mapping operations clearly may have an impact on the real-time performance of the cloud requests they are servicing, we propose to investigate three scenarios. In Scenario I (very conservative), we do not allow reconfiguring already established paths. In Scenario II, we only allow reconfiguring backup and/or synchronisation routes $\left(p^{\mathrm{B}}\right.$ and/or $\left.p^{\mathrm{S}}\right)$ for traffic that continues from one period to the next. In Scenario III, we assume complete freedom and thus also allow to change the primary paths $\left(p^{\mathrm{W}}\right)$. Yet, we always look for the optimal solution (in terms of minimal link bandwidth consumed on the PIP layer) with the lowest number of configuration changes.

\section{Optimization Model}

\subsection{Notations}

The cloud network is modeled by an undirected graph $G=(V, L)$ where $V$ is the node set (indexed by $v$ ) and $L$ is the link set (indexed by $\ell$ ), for which $\omega(v)$ denotes the set of links adjacent to $v$.

Traffic is defined by the number of service requests (demands), originating from a set of source/service nodes $V_{\mathrm{S}} \subseteq V$, with generic index $v_{\mathrm{S}}$. To simplify the exposure, we assume $V_{\mathrm{S}}=V$ from now on. Let $K$ be the set of service requests, indexed by $k . K$ is partitioned: $K=K^{\mathrm{ADD}} \cup K^{\mathrm{LEG}}$, where $K^{\mathrm{ADD}}$ are the new services to be granted and $K^{\mathrm{LEG}}$ the previously granted services which are still being served. 
Each service $k$ is characterized by its bandwidth requirement $\Delta_{k}$, its source (or origin) $v_{k}$, its datacenter resource requirement $R_{k}$, and $\delta_{k}$ (with $0 \leq \delta_{k} \leq 1$ ), representing the fraction of $\Delta_{k}$ that is required for synchronization between the primary and the backup data center.

Let $D \subseteq V$ be the set of data centers (indexed by $d$ ), and $\mathrm{CAPA}_{d}$ the resource capacity of data center $d$ (e.g., in terms of virtual machines). Let $n_{D}=|D|$ be the number of data centers. The current model assumes (at most) a single data center per node.

\subsection{Configurations}

The mathematical model we propose relies on the notion of configurations, where a configuration is associated with a set of service requests originating at a given source node. Let $C$ be the overall set of configurations: $C=\bigcup_{v \in V_{\mathrm{s}}} C_{v}$, where $C_{v}$ is the set of configurations associated with source node $v \in V_{\mathrm{s}}$. We define a configuration $c \in C_{v}$ by: (i) a set of 3 paths, one primary path $p^{\mathrm{w}}$ originating at $v_{s}$ towards a primary data center $d^{\mathrm{W}}$, one backup path $p^{\mathrm{B}}$ originating at $v_{s}$ towards a primary data center $d^{\mathrm{B}}$, and one synchronization paths $\left(p^{\mathrm{S}}\right)$ between the primary and the backup data center, as well as (ii) the service requests routed and protected by this set of 3 routes. We protect against single link failures as well as single data center failures.

More formally, in our mathematical model, a configuration is characterized by the given parameters:

- $p_{\ell, c}^{\mathrm{W}}$ (resp. $\left.p_{\ell, c}^{\mathrm{B}}\right)=1$ if link $\ell$ is used by the working (resp. backup) path of configuration $c, 0$ otherwise;

- $p_{\ell, c}^{\mathrm{s}}=1$ if link $\ell$ is used by the synchronization path of $c$ between the primary data center and the backup data center, 0 otherwise;

- $a_{v}^{\mathrm{W}, c}\left(\right.$ resp. $\left.a_{v}^{\mathrm{B}, c}\right)=1$ if node $v \in V_{\mathrm{D}}$ is selected as the primary (resp. backup) data center, 0 otherwise; For each link $\ell$, let $\beta_{\ell}^{\mathrm{W}}$ be the working bandwidth on $\ell, \beta_{\ell}^{\mathrm{B}}$ the backup bandwidth on $\ell$, and $\beta_{\ell}^{\mathrm{S}}$ the bandwidth of Synchronization path on $\ell$.

In the context of time-varying traffic, the set of configurations is partitioned as follows: $C=C^{\mathrm{ADD}} \cup C^{\mathrm{LEG}}$, where $C^{\mathrm{ADD}}$ is the set of newly generated configurations for the requests of $K^{\mathrm{ADD}}$.

$C^{\mathrm{LEG}}=C^{\mathrm{LEG} \_B S} \cup C^{\mathrm{LEG} \_\mathrm{W}}$ is the new set of selected configurations associated with $k \in K^{\mathrm{LEG}}$, with $C^{\mathrm{LEG} \_B S}$ being the configurations for which the working paths is unchanged, and $C^{\text {LEG_W }}$ the set of configurations in which at least the working path has been modified.

\subsection{Objective}

We first define the set of variables:

- $z_{k}^{c} \in\{0,1\}, c \in C^{\mathrm{ADD}}$ : decision variable that is equal to 1 if the configuration is selected for a given service $k, 0$ otherwise.

- $x_{k}^{\mathrm{LEG} \_\mathrm{BS}} \in\{0,1\}, k \in K^{\mathrm{LEG}}$ : decision variable that is equal to 1 if the protection or synchronization paths or the assigned DC of $k$ is modified (the working path is not modified).

- $x_{k}^{\mathrm{LEG}-\mathrm{W}} \in\{0,1\}, k \in K^{\mathrm{LEG}}$ : decision variable that is equal to 1 if the working path and possibly, the protection or synchronization paths or the assigned DC of $k$, is/are modified.

The objective function should take care of minimizing the overall (working + backup + synchronization) bandwidth requirements, and, in case of ties, and should encourage not to disturb the working paths as a first priority, and, as a second priority, not to disturb the backup/synchronization paths in case the same routing paths can be used for the legacy requests:

$$
\min \sum_{\ell \in L} \underbrace{\left(\beta_{\ell}^{\mathrm{W}}+\beta_{\ell}^{\mathrm{B}}+\beta_{\ell}^{\mathrm{S}}\right) \cdot\|\ell\|}_{\mathrm{BW}_{\ell}}+\text { PENAL }^{\text {DISRUPT_B }} \sum_{k \in K^{\text {LEG }}} x_{k}^{\text {LEE__BS }}+\text { PENAL }^{\text {DISRUPT_W }} \sum_{k \in K^{\text {LEG }}} x_{k}^{\text {LEG_W }}
$$

where $\|\ell\|$ represents the length of link $\ell$, PENAL ${ }^{\text {DISRUPT }-B}$ and PENAL ${ }^{\text {DISRUPT_w }}$ are weight penalty factors such that $\mathrm{PENAL}^{\text {DISRUPT_B }} \geq$ PENAL $^{\text {DISRUPT }}{ }_{-}$, and $\mathrm{BW}_{\ell}$ is total bandwidth requirement on a given link $\ell$.

Note that in Scenario I, only the first term of (1) is minimized, while the second plays a role only in Scenarios II and III, and the third term only for Scenario III.

\subsection{A Generic Model}

We next describe a generic model that encompasses all three scenarios. It relies on a decomposition scheme such that the master problem selects the best configurations out of a given set, while the pricing problem generates configurations, see Section 4 for more details. We next describe the master problem. 


$$
\begin{aligned}
& \sum_{c \in C_{v}} z_{k}^{c} \geq 1 \\
& \sum_{c \in C_{k}^{\mathrm{LG} \_ \text {BS }}} z_{k}^{c}=x_{k}^{\mathrm{LEG} \_ \text {BS }} \quad ; \quad x_{k}^{\mathrm{LEG} \_ \text {BS }} \leq 1-z_{k}^{\tilde{c}_{k}}
\end{aligned}
$$

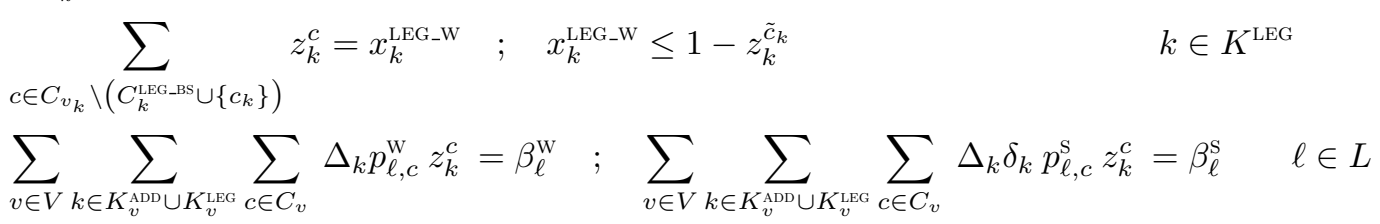

$$
\begin{aligned}
& \sum_{v \in V} \sum_{k \in K_{v}^{\mathrm{ADD}} \cup K_{v}^{\mathrm{LEG}}} \sum_{c \in C_{v}} \Delta_{k} p_{\ell^{\prime}, c}^{\mathrm{W}} p_{\ell, c}^{\mathrm{B}} z_{k}^{c} \leq \beta_{\ell}^{\mathrm{B}} \\
& \sum_{v \in V} \sum_{k \in K_{v}^{\mathrm{ADD}} \cup K_{v}^{\mathrm{LEG}}} \sum_{c \in C_{v}} \Delta_{k} a_{v}^{\mathrm{W}, c} p_{\ell, c}^{\mathrm{B}} z_{k}^{c} \leq \beta_{\ell}^{\mathrm{B}} \\
& \ell^{\prime} \in L, \ell \in L \backslash\left\{\ell^{\prime}\right\} \\
& v \in V_{\mathrm{D}}, \ell \in L \\
& \sum_{v \in V} \sum_{k \in K_{v}^{\mathrm{ADD}} \cup K_{v}^{\mathrm{LEG}}} \sum_{c \in C_{v}} R_{k}\left(a_{v}^{\mathrm{W}, c}+a_{v}^{\mathrm{B}, c}\right) z_{k}^{c} \leq \mathrm{CAPA}_{v} \\
& z_{k}^{c} \in\{0,1\} \quad c \in C, k \in K ; \quad \beta_{\ell}^{\mathrm{W}}, \beta_{\ell}^{\mathrm{B}}, \beta_{\ell}^{\mathrm{s}} \in \mathbb{R} \quad \ell \in L \\
& x_{k}^{\mathrm{LEG} \_\mathrm{BS}}, x_{k}^{\mathrm{LEG} \_\mathrm{W}} \in\{0,1\} \\
& k \in K_{v}^{\mathrm{ADD}}, v \in V \\
& k \in K^{\mathrm{LEG}} \\
& v \in V_{\mathrm{D}} \\
& k \in K^{\mathrm{LEG}} \text {. }
\end{aligned}
$$

Constraints (2) take care of granting every new services. Constraints (3) (left equality) detect the legacy services with an identical working path, but a different backup or synchronization path. Constraints (4) (left equality) detect the legacy services with a different working path, and potentially a different backup or synchronization path. Constraints (3) (right equality) and (4) ensure consistency between the values of $z_{k}^{\tilde{c_{k}}}$ and $x_{k}^{\mathrm{LEG}-\mathrm{W}}$, and between the values of $z_{k}^{\tilde{c}_{k}}$ and $x_{k}^{\mathrm{LEG}-\mathrm{BS}}$ respectively. Constraints (4) (right equality) forbid $x_{k}^{\text {LEG-W }}=x_{k}^{\text {LEG_BS }}=1$, i.e., if only the backup (or the synchronization) path is changed, then we cannot change the primary path without being inconsistent. Constraints (5) and (6) compute the working, synchronization and backup bandwidth requirements, respectively. Constraints (7) take care of the backup bandwidth requirement in order to be protected against a single data center failure. Constraints (7) guarantee sufficient backup bandwidth $\ell$ to handle any data center failure. Constraints (8) check that the capacity $\left(\mathrm{CAPA}_{v}\right)$ of the data centers $\left(v \in V_{\mathrm{D}}\right)$ is not exceeded. The last three set of constraints, i.e., (9) and (10), define the domain of the variables.

\section{Solution Scheme}

The above master problem (RMP) is solved iteratively, alternating with the solution of the pricing problem (PP) that determines augmenting configurations, i.e., routes for $\mathrm{W}, \mathrm{B}$ and $\mathrm{s}$ paths such that their addition to the restricted master problem entails an improvement of the optimal value of the current restricted master problem, see the flowchart in Fig. 2. Each PP is written for a given source node $v_{k}$ and for a given request originating at $v_{k}$. Parameters $\Delta_{k}$ and $\delta_{k}$ retain their definition for a request $k$ as in the RMP.

The sets of variables corresponds to the set of parameters in the master problem, i.e., $p_{\ell}^{\mathrm{W}}, p_{\ell}^{\mathrm{B}}, p_{\ell}^{\mathrm{S}}$, $a_{v}^{\mathrm{W}}, a_{v}^{\mathrm{B}}, d_{v}^{\mathrm{W}}, d_{v}^{\mathrm{B}}$ and $d_{v}^{\mathrm{s}}$. We need to distinguish two pricing problems, one for the requests newly added in the considered period (ADD), another one

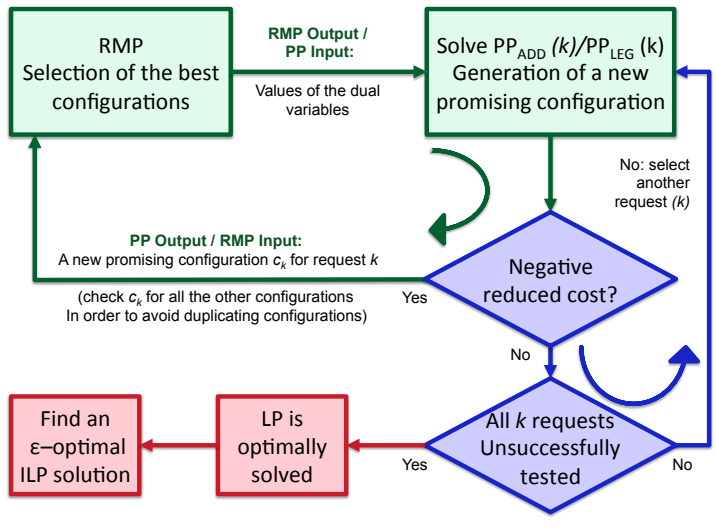

Fig. 2: Solution Process for the legacy requests that remain from the previous period (LEG). We first discuss $\mathrm{PP}_{\mathrm{ADD}}$. 
While the pricing problem is solved for a given request, say $k$, we check whether it allows to generate augmenting configurations for all the requests with the same origin node as $k$. In other words, if an augmenting configuration is found for $k$, we check whether it is also an augmenting configuration for any $k^{\prime}$ from the same source, i.e., for which $v_{k^{\prime}}=v_{k}$. If it is the case, we add $c_{k}$ to the set of configurations associated with $k^{\prime}$. Once we have examined all $k^{\prime}$ such that $v_{k^{\prime}}=v_{k}$, the next PP is solved for a request with a different source node. This way, instead of a number of iterations in the order of the number of requests - which can be quite computationally expensive - we expect a complete round to be in the order of the number of source nodes.

For a given source node, requests are initially ordered according to decreasing bandwidth requirements, but we examine in a round robin fashion from one round to the next, in order not to always start solving the pricing problem for the same request.

The objective of $\mathrm{PP}_{\mathrm{ADD}}(k)$ with $k \in K^{\mathrm{ADD}}$ is to minimize the reduced cost $\overline{\operatorname{COST}}^{\mathrm{ADD}}\left(z_{k}\right)$, which is straightforwardly derived from the RMP — see [9] if not familiar with linear programming tools. Note that two quadratic terms appear in the reduced cost (i.e., those associated with the dual variables of (6) and (7)), but they can be easily linearized through the introduction of two sets of binary variables $p_{\ell \ell^{\prime}}^{\mathrm{WB}}$ and $p_{v \ell}^{\mathrm{WB}}$ and the addition of the following constraints, for all $\ell^{\prime} \in L, \ell \in L \backslash\left\{\ell^{\prime}\right\}, v \in V_{\mathrm{D}}$ :

$$
p_{\ell \ell^{\prime}}^{\mathrm{WB}} \leq p_{\ell^{\prime}}^{\mathrm{W}} \quad ; \quad p_{\ell \ell^{\prime}}^{\mathrm{WB}} \leq p_{\ell}^{\mathrm{B}} \quad ; \quad p_{\ell \ell^{\prime}}^{\mathrm{WB}} \geq p_{\ell^{\prime}}^{\mathrm{W}}+p_{\ell^{\prime}}^{\mathrm{W}}-1 \quad ; \quad p_{v \ell}^{\mathrm{WB}} \leq a_{v}^{\mathrm{W}} \quad ; \quad p_{v \ell}^{\mathrm{WB}} \leq p_{\ell}^{\mathrm{B}} \quad ; \quad p_{v \ell}^{\mathrm{WB}} \geq a_{v}^{\mathrm{W}}+p_{\ell^{\prime}}^{\mathrm{W}}-1 .
$$

To complete the PP, we need to enforce that the path and data center variables obey the following constraints $^{1}$ : the flow constraints for the undirected working, backup and synchronization paths, the link disjointness constraints of paths $p_{\mathrm{W}}$ and $p_{\mathrm{B}}$, and the fact that each configuration has exactly one primary and one back up data center, while primary and backup data centers are different.

Now, for the legacy constraints, we have a slightly different $\mathrm{PP}_{\mathrm{LEG}}$. In short, the only change is that for these requests, the path changes that we can make are restricted, depending on the scenario (recall Section 2.3). In Scenario I, the full routing of these requests is given from the first period, so we do not need to solve any PP for them. In Scenario II, we cannot change the working path and thus the corresponding values $\tilde{p}_{\ell}^{W}$ are given a priori, while the other paths follow similar constraints as in $\mathrm{PP}_{\mathrm{ADD}}$. For Scenario III, we have the full flexibility and thus the PP is the same as in the ADD case. In all cases, for $k \in K^{\mathrm{LEG}}$, the expression of $\overline{\mathrm{COST}}^{\mathrm{LEG}}\left(z_{k}\right)$ is easily derived from $\overline{\operatorname{COST}}^{\mathrm{ADD}}\left(z_{k}\right)$.

\section{Numerical Results}

\subsection{Data Sets}

We used the USA network illustrated in Fig. 3, which we divided into three regions, each with their own traffic pattern. Each request is randomly generated with a bandwidth requirement $\left(\Delta_{k}\right)$ normalized between 0 and 1 , and a $\delta_{k}=0.1$ synchronization factor. We assume 4 data centers, located in CA (node 5), WY(node 6), TX (node 13), OH (node 15). We consider the transition from one period to a next, where the eventual number of requests varies from 20 to 80 requests. While this total remains the same among the two successive periods, the geographical distribution of their origins changes from the first to the second period. Initially, i.e., during the first planning time period, the traffic is distributed as follows: $30 \%$ in region $1,50 \%$ in region 2 , and $20 \%$ in region 3 . For each of those regions, sources are uniformly randomly spread over the nodes within the respective region. Next, during the second time period, the traffic is randomly modified (again uniformly distributed over nodes within each region): the traffic of period 1 that continues into period 2, designated as legacy traffic, varies per region as indicated in Table 1. We consider three scenarios, where the overal legacy traffic (over all three regions together) is set to $40 \%, 60 \%$, and $80 \%$ respectively.

\subsection{Bandwidth Requirements with Time-varying Traffic}

We conducted experiments under different traffic loads and computed the bandwidth requirements under different backup provisioning scenarios as described in Section 2.3. For each traffic load, results correspond to averages over 5 data instances. The observations from the results plotted in Fig. 4 are as follows:

\footnotetext{
${ }^{1}$ Since these constraints are straightforward to write down for anyone familiar with linear programming, we omit the exact mathematical formulas.
} 


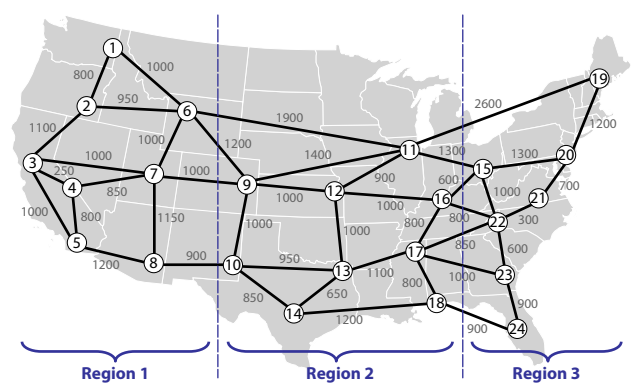

Fig. 3: USA network with its different regions.
Table 1: Traffic change distribution over the three regions, where the change is expressed in $\%$ of the total traffic volume summed over all regions.

\begin{tabular}{l||c|c|c} 
Scenario & Region 1 & Region 2 & Region 3 \\
\hline \hline $40 \%$ & $20 \%$ DROP & $30 \%$ DROP & $10 \%$ DROP \\
legacy & $20 \%$ ADD & $10 \%$ ADD & $30 \%$ ADD \\
\hline $60 \%$ & $10 \%$ DROP & $20 \%$ DROP & $10 \%$ DROP \\
legacy & $10 \%$ ADD & $10 \%$ ADD & $20 \%$ ADD \\
\hline $80 \%$ & $10 \%$ DROP & $10 \%$ DROP & - \\
legacy & $10 \%$ ADD & - & $10 \%$ ADD
\end{tabular}
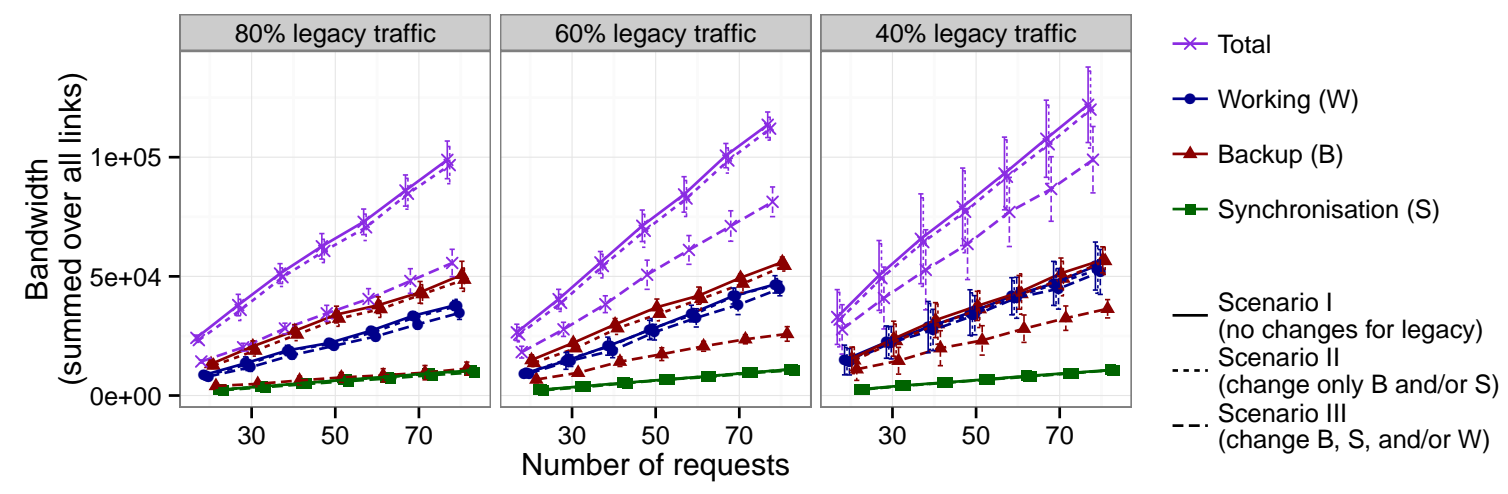

Fig. 4: Bandwidth requirements.

- Bandwidth savings are not significant as long as we do not allow disruption, i.e., reconfiguration, of some working paths (from Scenario I to II the cost difference is not big, but for Scenario III it is).

- Bandwidth savings mainly stem from the reduction of backup capacity (for all three legacy traffic cases).

- The bandwidth for the synchronization among primary and backup data centers (the $p^{\mathrm{S}}$ ), even if provisioned on different paths, is rather constant for all three reconfiguration scenarios.

- The achieved bandwidth savings (for Scenario III), relative to the total cost, decreases with decreasing the fraction of legacy traffic (obviously, since the savings come from changing legacy requests, if there are fewer of them then the saving potential diminishes).

Now, one may wonder what the amount of path reconfigurations is that is required to achieve those bandwidth savings (esp. for Scenario III). This we have summarized in Table 2. This shows that in Scenario III, even though more than half of the legacy requests need to have some of their paths reconfigured, only around $20 \%$ of them require a change of the working path. Thus, if we may assume that a sufficient fraction of the traffic does not have QoS requirements that prohibit path reconfiguration, this may be an acceptable solution to cut down the VNet operating costs in terms of needed PIP resources.

\section{Conclusion}

We investigated the interest of re-provisioning the working and the backup paths in the context of anycast routing traffic in cloud computing, assuming time-varying traffic, where the path provisioning can be updated periodically. While it seems that periodic backup reconfiguration is only advantageous if we reconfigure some working paths as well, further experiments should evaluate the impact of the server locations (e.g., scattered vs. paired as in [10]), and investigate different time-varying traffic patterns. 
Table 2: Number of requests that need reconfigurations $(\mathrm{W}=$ of the working path, $\mathrm{B}=$ of the backup path, $\mathrm{S}=$ of the synchronisation path). The last column shows the fraction of modified legacy requests $\left(\alpha_{\mathrm{LEG}}\right)$, averaged over all demand instances.

\begin{tabular}{|c|c|c|c|c|c|c|c|c|c|}
\hline \multirow{2}{*}{$\begin{array}{l}\text { Legacy } \\
\text { traffic }\end{array}$} & \multirow{2}{*}{ Path changes } & \multicolumn{7}{|c|}{ Total demand (number of requests) } & \multirow{2}{*}{$\begin{array}{c}\alpha_{\mathrm{LEG}} \text { over } \\
\text { all demands }\end{array}$} \\
\hline & & 20 & 30 & 40 & 50 & 60 & 70 & 80 & \\
\hline \multirow{4}{*}{$80 \%$} & Scen. II - Changed B and/or S & 9.6 & 12.8 & 16.6 & 17.2 & 20.6 & 22.6 & 22.8 & $46.73 \%$ \\
\hline & Scen. III - Fixed W, changed B/S & 6.2 & 8.2 & 12.4 & 16.8 & 16.2 & 18.0 & 25.6 & $37.08 \%$ \\
\hline & Scen. III - Changed W & 3.2 & 3.8 & 6.8 & 5.6 & 10.0 & 12.8 & 7.8 & $18.14 \%$ \\
\hline & Scen. III - Total changes & 9.4 & 12.0 & 19.2 & 22.4 & 26.2 & 30.8 & 33.4 & $55.22 \%$ \\
\hline \multirow{4}{*}{$60 \%$} & Scen. II - Changed B and/or S & 8.0 & 10.0 & 12.2 & 15.6 & 17.4 & 19.6 & 15.2 & $50.25 \%$ \\
\hline & Scen. III - Fixed W, changed B/S & 4.8 & 6.2 & 8.8 & 12.2 & 11.4 & 11.8 & 16.0 & $34.98 \%$ \\
\hline & Scen. III - Changed W & 3.0 & 3.0 & 5.8 & 6.0 & 9.4 & 11.4 & 6.6 & $21.83 \%$ \\
\hline & Scen. III - Total changes & 7.8 & 9.2 & 14.6 & 18.2 & 20.8 & 23.2 & 22.6 & $56.82 \%$ \\
\hline \multirow{4}{*}{$40 \%$} & Scen. II - Changed B and/or S & 5.8 & 7.0 & 9.4 & 12.2 & 10.6 & 13.4 & 11.6 & $54.12 \%$ \\
\hline & Scen. III - Fixed W, changed B/S & 3.0 & 3.6 & 6.8 & 7.4 & 7.2 & 7.6 & 11.2 & $34.16 \%$ \\
\hline & Scen. III - Changed W & 1.8 & 2.4 & 4.2 & 3.6 & 5.8 & 6.0 & 4.4 & $20.87 \%$ \\
\hline & Scen. III - Total changes & 4.8 & 6.0 & 11.0 & 11.0 & 13.0 & 13.6 & 15.6 & $55.03 \%$ \\
\hline
\end{tabular}

\section{ACKNOWLEDGMENT}

B. Jaumard has been supported by a Concordia University Research Chair (Tier I) and by an NSERC (Natural Sciences and Engineering Research Council of Canada) grant. D. Medhi has been supported by National Science Foundation Grant No. CNS-1217736.

\section{REFERENCES}

[1] X. He and G.-S. Poo, "Sub-reconfiguration of backup paths based on shared path protection for WDM networks with dynamic traffic pattern," in Proc. 9th Int. Conf. Commun. Systems (ICCS), Sep. 2004, pp. 391-395.

[2] S. Srivastava, S. R. Thirumalasetty, and D. Medhi, "Network traffic engineering with varied levels of protection in the next generation internet," in Performance Evaluations and Planning Methods for the Next Generation Internet, A. Girard, B. Sanso, and F. Vazquez-Abad, Eds. Springer Verlag, 2005.

[3] S. Sebbah and B. Jaumard, "Differentiated quality-of-protection in survivable wdm mesh networks using p-structures," Computer Commun., vol. 36, pp. 621-629, Mar. 2013.

[4] C. Develder, J. Buysse, B. Dhoedt, and B. Jaumard, "Joint dimensioning of server and network infrastructure for resilient optical grids/clouds," IEEE/ACM Trans. Netw., pp. 1-16, Oct. 2013.

[5] M. Bui, B. Jaumard, and C. Develder, "Anycast end-to-end resilience for cloud services over virtual optical networks (invited)," in Proc. 15th Int. Conf. Transparent Optical Netw. (ICTON), Cartagena, Spain, 23-27 Jun. 2013.

[6] C. Develder, M. De Leenheer, B. Dhoedt, M. Pickavet, D. Colle, F. De Turck, and P. Demeester, "Optical networks for grid and cloud computing applications," Proc. IEEE, vol. 100, no. 5, pp. 1149-1167, May 2012.

[7] N. Chowdhury and R. Boutaba, "A survey of network virtualization," Computer Netw., vol. 54, pp. 862-876, Apr. 2010.

[8] P. Primet Vicat-Blanc, S. Soudan, and D. Verchere, "Virtualizing and scheduling optical network infrastructure for emerging IT services," J. Optical Commun. Netw., vol. 1, pp. A121-A132, 2009.

[9] V. Chvatal, Linear Programming. Freeman, 1983.

[10] M. Bui, B. Jaumard, and C. Develder, "Resilience options for provisioning anycast cloud services with virtual optical networks," in Proc. IEEE Int. Conf. on Commun. (ICC), Sydney, Australia, 10-14 Jun. 2014. 